\title{
Basic Study on RF Characteristics of Thin-Film Transmission Line Employing ML/CPW Composite Structure on Silicon Substrate and Its Application to a Highly Miniaturized Impedance Transformer
}

\author{
Jang-Hyeon Jeong, Ki-Jun Son, and Young Yun ${ }^{\dagger}$ \\ Department of Communication Engineering, Korea Maritime and Ocean University, Busan 606-791, Korea
}

Received July 28, 2014; Revised August 5, 2014; Accepted August 25, 2014

\begin{abstract}
A thin-film transmission line (TFTL) employing a microstrip line/coplanar waveguide (ML/CPW) was fabricated on a silicon substrate for application to a miniaturized on-chip RF component, and the RF characteristics of the device with the proposed structure were investigated. The TFTL employing a ML/CPW composite structure exhibited a shorter wavelength than that of a conventional coplanar waveguide and that of a thin-film microstrip line. When the TFTL with the proposed structure was fabricated to have a length of $\lambda / 8$, it showed a loss of less than $1.12 \mathrm{~dB}$ at up to $30 \mathrm{GHz}$. The improvement in the periodic capacitance of the TFTL caused for the propagation constant, $\beta$, and the effective permittivity, $\varepsilon_{\text {eff }}$, to have values higher than those of a device with only a conventional coplanar waveguide and a thin film microstrip line. The TFTL with the proposed structure showed a $\beta$ of $0.53 \sim 2.96 \mathrm{rad} / \mathrm{mm}$ and an $\varepsilon_{\text {eff }}$ of $22.3 \sim 25.3$ when operating from 5 to $30 \mathrm{GHz}$. A highly miniaturized impedance transformer was fabricated on a silicon substrate using the proposed TFTL for application to a low-impedance transformation for broadband. The size of the impedance transformer was $0.01 \mathrm{~mm} 2$, which is only $1.04 \%$ of the size of a transformer fabricated using a conventional coplanar waveguide on a silicon substrate. The impedance transformer showed excellent RF performance for broadband.
\end{abstract}

Keywords: Thin-film transmission line, Microstrip line/coplanar waveguide (ML/CPW) composite structure, Silicon, Impedance transformer, RFIC

\section{INTRODUCTION}

Recently, wireless communication system have come to demand the development of a communication systems that have a low-cost and are compact in size [1-8]. However, conventional passive RF components have been fabricated outside of the RFIC due to their bulky size. Therefore, the miniaturization of passive components is a critical issue for wireless communica-

${ }^{\dagger}$ Author to whom all correspondence should be addressed: E-mail: yunyoung@kmou.ac.kr

Copyright $(2015$ KIEEME. All rights reserved. This is an open-access article distributed under the terms of the Creative Commons Atribution Non-Commercial
License (httr:///creativecommons.org/licenses/by-nc/3.0) which permits unrestricted noncommercial use.

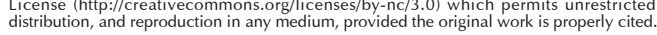

tion systems. Thin-film transmission lines have been proposed in order to reduce the size of the circuitry on a semiconducting substrate [9-13], and according to our previous report [11], a thin-film transmission line (TFTL) employing a microstrip line/ coplanar waveguide (ML/CPW) composite structure showed a shorter wavelength than that showed by conventional thin film transmission lines. The basic characteristics of the TFTL with the composite structure should be thoroughly explored in order to apply it to various RF components on a semiconducting substrate. However, an extensive study of the basic characteristics of the TFTL employing an ML/CPW has not yet been performed.

In this work, we use a theoretical and an experimental analysis to investigate the basic characteristics of the TFTL employing a 
ML/CPW composite structure for application to the miniaturization of passive RF components in a radio frequency integrated circuit (RFIC). The results indicate that the TFTL with a ML/CPW composite structure presented a shorter wavelength than that of conventional transmission lines, which therefore enables the realization of highly miniaturized passive on-chip components for RFIC. The TFTL with a ML/CPW composite structure was used to achieve a highly miniaturized impedance transformer fabricated on silicon substrate. The size of the impedance transformer was of $0.01 \mathrm{~mm}^{2}$, which is $1.04 \%$ of the size of a transformer fabricated using a conventional coplanar waveguide on a silicon substrate.

\section{STRUCTURE OF THE THIN-FILM TRANSMISSION LINE (TFTL) EMPLOYING MICROSTRIP LINE/COPLANAR WAVEGUIDE (ML/CPW) COMPOSITE STRUCTURE}

Figure 1 shows the structure of a conventional transmission lines and of a thin-film microstrip line $[9,10]$. As shown in Fig 1 (c), for the thin-film microstrip line, the second ground plane is placed on the silicon substrate, and a $\mathrm{SiO}_{2}$ thin-film exists between the second ground and the signal line on the top layer. Compared to a conventional microstrip line without a second ground plane and a coplanar waveguide, the thin-film microstrip line has a high periodic coupling capacitance, $\mathrm{C}_{\mathrm{b}}$, due to a tight coupling between the signal line and the second ground plane. Therefore, the thin-film microstrip line showed a much shorter wavelength than that of a conventional microstrip line and a coplanar waveguide because the wavelength is inversely proportional to the periodic capacitance of the transmission line as follows:

$$
\lambda_{g}=\frac{1}{f \sqrt{L C}}
$$

In this work, we propose the use of a thin-film transmission line (TFTL) that further reduces the wavelength by employing a microstrip line/coplanar waveguide (ML/CPW) composite structure, as shown in Fig. 2. As can be seen in this figure, the second ground plane is placed on the silicon substrate, and a $\mathrm{SiO}_{2}$ thin-film exists between the second ground and the signal line on the top layer. In addition, the ground planes are placed on both sides of the signal line, which were connected to the second ground plane through the contacts. When compared to a thin-film microstrip line, the proposed structure has an additional capacitance, $\mathrm{C}_{\mathrm{a}}$, due to a coupling between the signal line and the top layer ground planes, and the total periodic shunt capacitance of $C_{a}+C_{b}$ further reduces the wavelength.

\section{PERIODIC CAPACITANCE OF THE TFTL EMPLOYING ML/CPW COMPOSITE STRUCTURE}

Basic RF parameters of microwave transmission lines are well known to be expressed by a periodic capacitance and an inductance of an equivalent LC circuit [14]. Therefore, we extracted the equivalent periodic capacitance from the TFTL by employing a ML/CPW structure and the conventional meander line. For a
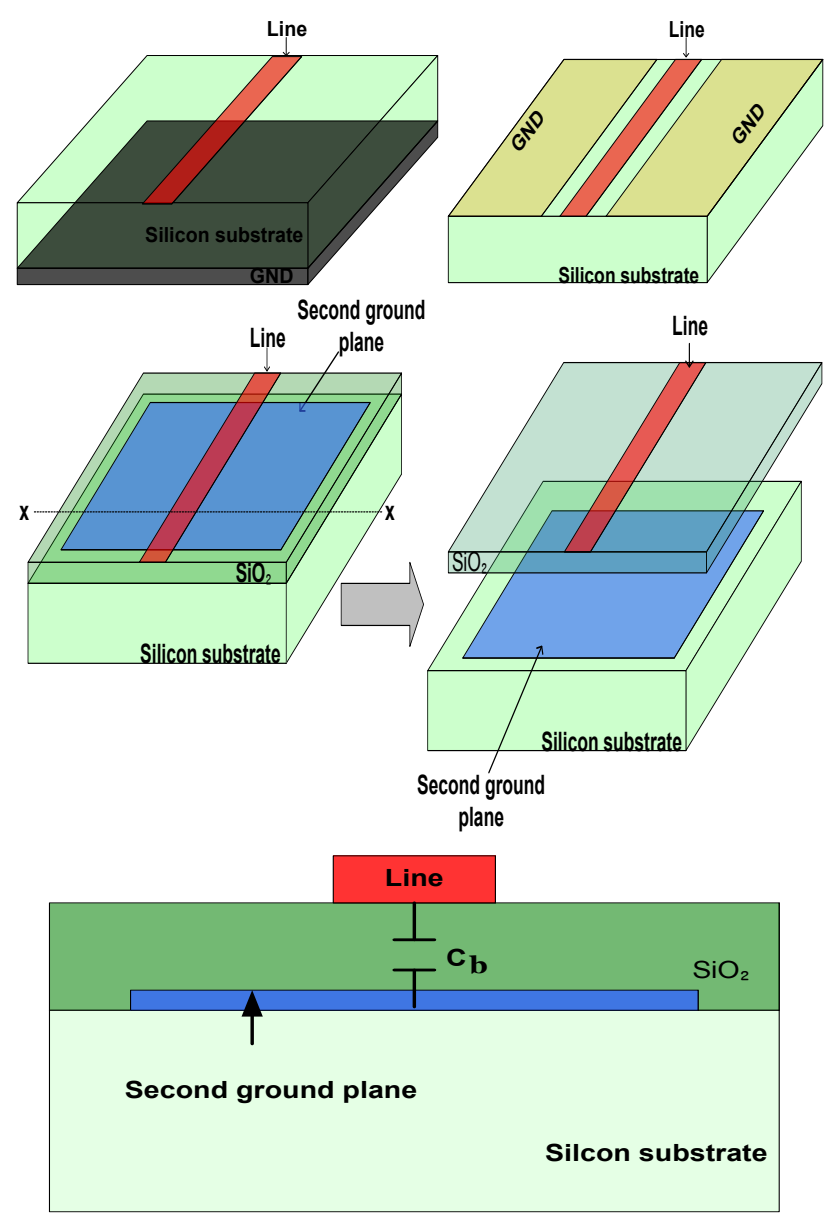

Fig. 1. Structure of a conventional transmission line: (a) microstrip line, (b) coplanar waveguide, and (c) thin-film microstrip line.
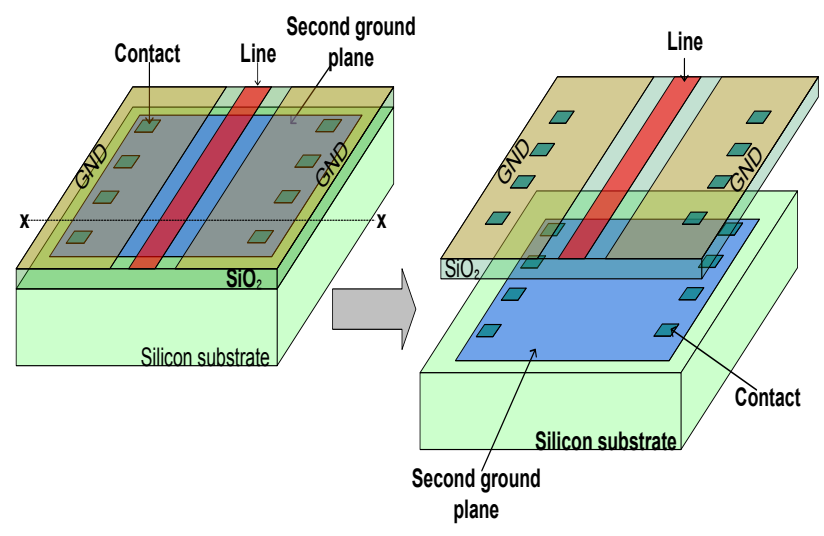

(A cross-sectional view of $\mathrm{X}-\mathrm{X}$ )

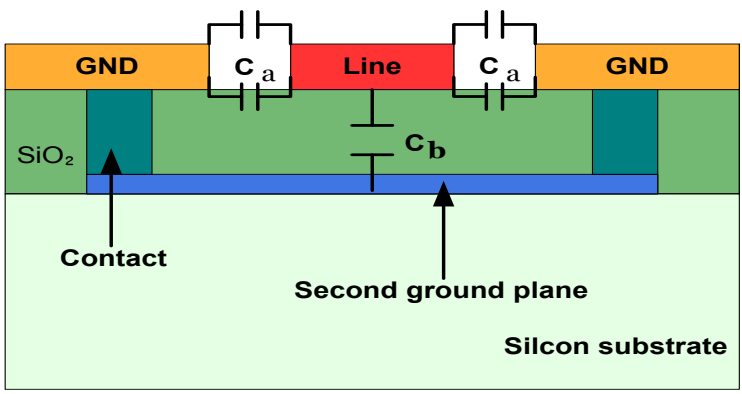

Fig. 2. The thin-film transmission line (TFTL) employing a microstrip line/coplanar waveguide (ML/CPW) composite structure. 


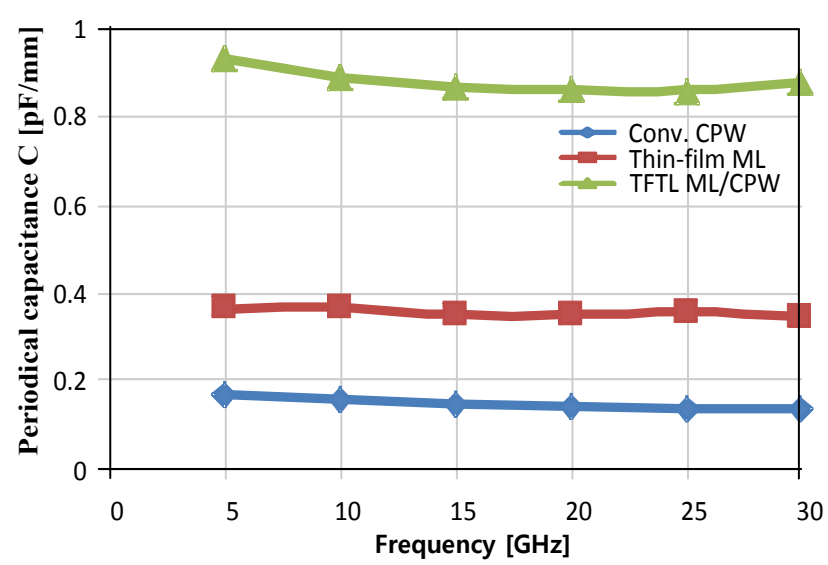

Fig. 3. Measured equivalent periodic capacitance per unit length for various transmission lines on a silicon substrate.

low-loss transmission line, the propagation constant, $\beta$, and the characteristic impedance, $Z_{0}$, are given as [14]

$$
\begin{aligned}
& \beta \approx \omega \sqrt{L C} \\
& Z_{0}=\sqrt{\frac{L}{C}}
\end{aligned}
$$

Using the above equations, we can obtain the following result.

$$
C=\frac{L}{Z_{0}^{2}}=\frac{\beta}{\omega Z_{0}}=\frac{1}{\omega Z_{0}} \cdot \frac{2 \pi}{\lambda}
$$

Figure 3 shows the equivalent periodic capacitance of the various transmission lines on the silicon substrate. The TFTL employing the ML/CPW composite structure was fabricated on a silicon substrate at a height of $600 \mu \mathrm{m}$, and the $\mathrm{SiO}_{2}$ thinfilm has a thickness of $1 \mu \mathrm{m}$. The signal line width is $20 \mu \mathrm{m}$, and the distance between the signal line and the ground plane is 20 $\mu \mathrm{m}$. As shown in this figure, the TFTL with a ML/CPW composite structure presents a much higher capacitance than that of a conventional coplanar waveguide and a thin-film microstrip line. Concretely, the proposed composite structure shows a capacitance value of $0.88 \sim 0.93 \mathrm{pF} / \mathrm{mm}$ from 5 to $30 \mathrm{GHz}$, while a conventional coplanar waveguide and thin-film microstrip line show a capacitance value of $0.13 \sim 0.17$ and $0.34 \sim 0.36 \mathrm{pF} / \mathrm{mm}$, respectively, for the same frequency range.

\section{RF CHARACTERISTICS OF THE TFTL EMPLOYING ML/CPW COMPOSITE STRUCTURE}

Figure 4 shows the wavelengths for various transmission lines. The TFTL employing a ML/CPW composite structure was fabricated on a silicon substrate at a height of $600 \mu \mathrm{m}$, and the $\mathrm{SiO}_{2}$ thin-film had a thickness of $1 \mu \mathrm{m}$. The signal line width is $20 \mu \mathrm{m}$,

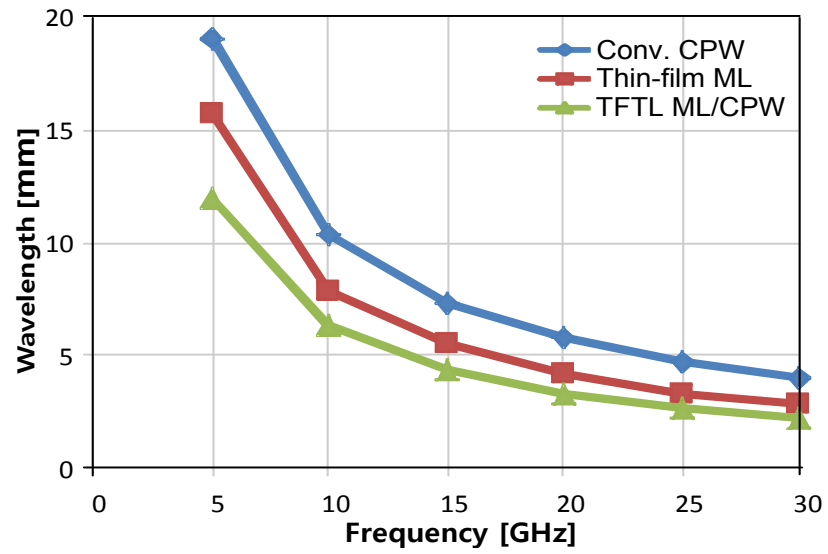

Fig. 4. Measured wavelength of various transmission lines on silicon substrate.

Table 1. Measured insertion loss of conventional coplanar waveguide and of the TFTL employing a ML/CPW composite structure on a silicon substrate with a length of $\lambda / 8$.

\begin{tabular}{ccccc}
\hline Items & $15 \mathrm{GHz}$ & $20 \mathrm{GHz}$ & $25 \mathrm{GHz}$ & $30 \mathrm{GHz}$ \\
\hline $\begin{array}{c}\text { Conventional } \\
\mathrm{CPW}\end{array}$ & $1.34 \mathrm{~dB}$ & $1.27 \mathrm{~dB}$ & $1.27 \mathrm{~dB}$ & $1.08 \mathrm{~dB}$ \\
\hline $\begin{array}{c}\text { TFTL } \\
\text { ML/CPW }\end{array}$ & $1.12 \mathrm{~dB}$ & $0.92 \mathrm{~dB}$ & $0.75 \mathrm{~dB}$ & $0.65 \mathrm{~dB}$ \\
\hline
\end{tabular}

and the distance between the signal line and the ground plane is of $20 \mu \mathrm{m}$. When compared to a conventional coplanar waveguide and a thin-film microstrip line, the proposed composite structure shows a shorter wavelength due to its higher periodic capacitance. Specifically, at $10 \mathrm{GHz}$, the wavelengths for the conventional coplanar waveguide and of the thin film waveguide are 10.35 and $7.83 \mathrm{~mm}$, respectively, while the wavelength of the TFTL with a ML/CPW composite structure is $6.26 \mathrm{~mm}$, which is $60.5 \%$ that of a conventional coplanar waveguide.

In this work, we have compared the loss of the proposed composite structure with that of a conventional coplanar waveguide. To provide a fair comparison of the loss, the loss of the proposed composite structure and that of the conventional coplanar waveguide of the same electrical length should be compared to each other because the proposed composite structure shows a much shorter wavelength than that of the conventional coplanar waveguide. Therefore, the loss of the proposed composite structure and that of the conventional coplanar waveguide with a length of $\lambda / 8$ were compared to each other, and the results are shown in Table. 1. As shown in this table, the TFTL employing the ML/CPW composite structure presented less loss than the conventional coplanar waveguide. The lower level of loss of the proposed composite structure originates from the tight coupling between the signal line and the second ground plane. In the case of a conventional coplanar waveguide on a silicon substrate, some of the RF current flows from the signal line to top layer ground plane through the silicon substrate due to a relatively high conductivity of the silicon substrate, which causes a relatively high loss of electromagnetic energy [15]. In the case of the proposed composite structure, however, there is no RF current flowing from the signal line to the top layer ground plane due to the presence of the $\mathrm{SiO}_{2}$ insulation film, and most of the electromagnetic energy exists between the signal line and second ground plane due to the tight coupling, which prevents the loss of electromagnetic 
energy.

Figure 5 shows the propagation constant, $\beta$, of the various transmission lines on the silicon substrate. As shown in this figure, the TFTL employing the ML/CPW composite structure shows a much higher $\beta$ than a conventional coplanar waveguide and a thin-film microstrip line. Specifically, the proposed composite structure shows a $\beta$ of $0.53 \sim 2.96 \mathrm{rad} / \mathrm{mm}$ from 5 to $30 \mathrm{GHz}$, while the coplanar waveguide and thin-film microstrip line show a $\beta$ of $0.33 \sim 1.57 \mathrm{rad} / \mathrm{mm}$ and $0.4 \sim 2.27 \mathrm{rad} / \mathrm{mm}$, respectively, for the same frequency range. Eq. (2) shows that the higher periodic capacitance is an indication of a higher $\beta$. Therefore, the higher $\beta$ of the proposed composite structure originates from the higher periodic capacitance.

Figure 6 shows the effective permittivity, $\varepsilon_{\text {eff }}$, of the various transmission lines on a silicon substrate. The $\varepsilon_{\text {eff }}$ was extracted using the following equation

$$
\varepsilon_{\text {eff }}=\left(\frac{2 \pi}{\omega \lambda} \cdot \frac{1}{\sqrt{\varepsilon_{0} \mu_{0}}}\right)^{2}
$$

where, $\omega, \lambda, \varepsilon_{0}$, and $\mu_{0}$ are the angular frequency, wavelength, and permittivity and permeability of air, respectively. As shown in this figure, the TFTL with a ML/CPW composite structure shows an effective permittivity that is much higher than that of conventional transmission lines. Specifically, the TFTL with the composite structure shows an $\varepsilon_{\text {eff }}$ of $22.3 \sim 25.3$ from 5 to $30 \mathrm{GHz}$ while the conventional coplanar waveguide and the thin-film microstrip line shows an $\varepsilon_{\text {eff }}$ of 6.28 9.87 and 13.0 14.6, respectively, over the same frequency range. The higher $\varepsilon_{\text {eff }}$ of the proposed composite structure originates from the higher periodic capacitance, which can be explained through the following equations in which the propagation constant, $\beta$, for a non-magnetic substrate is given by

$$
\beta \approx \omega \sqrt{L C}=\omega \sqrt{\mu_{0} \varepsilon_{0} \varepsilon_{e f f}}
$$

The above equation leads to the following result

$$
\varepsilon_{e f f}=\frac{\sqrt{L C}}{\mu_{0} \varepsilon_{0}}
$$

where we can see that a higher periodic capacitance indicates a higher $\varepsilon_{\text {eff }}$. As shown in Fig. 4, an increase in the effective permittivity of the proposed composite structure resulted in a reduction of the wavelength, and the above results indicate that the TFTL with a ML/CPW composite structure is favorable for use in the miniaturization of RF components.

\section{A HIGHLY MINIATURIZED THIN FILM IMPEDANCE TRANSFORMER EMPLOYING ML/CPW COMPOSITE STRUCTURE}

We used the TFTL with a ML/CPW composite structure to fabricate a highly miniaturized impedance transformer on a

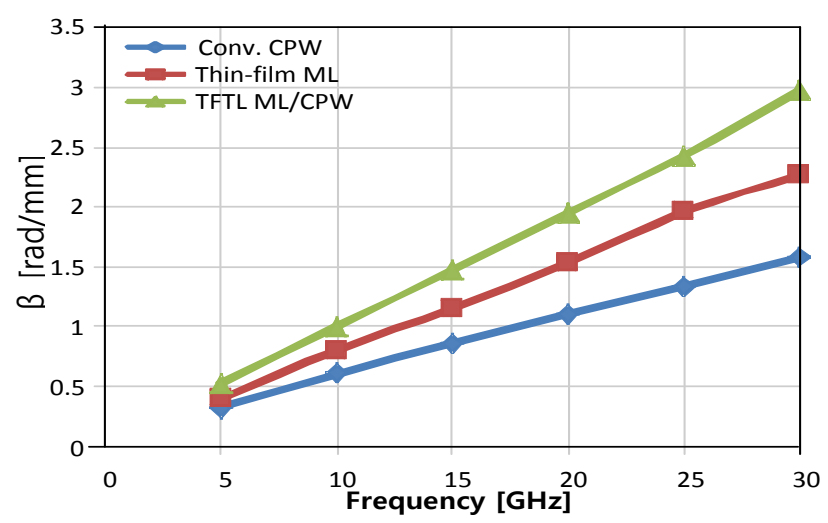

Fig. 5. Measured propagation constant of various transmission lines on a silicon substrate.

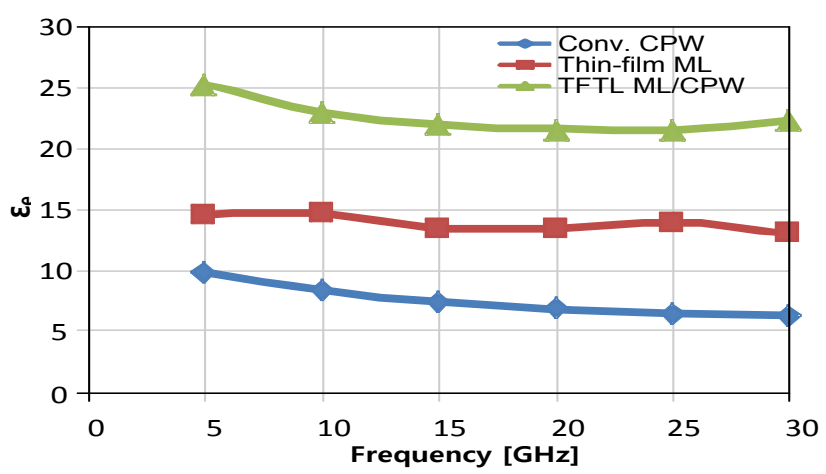

Fig. 6. Measured effective permittivity of various transmission lines on a silicon substrate.

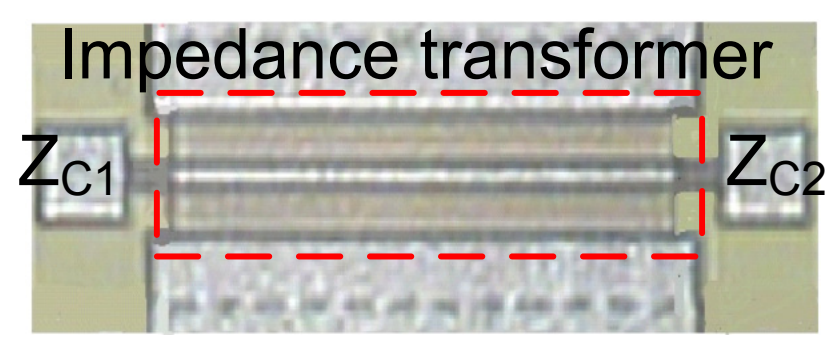

Fig. 7. A photograph of the miniaturized thin film impedance transformer employing an ML/CPW composite structure.

Table 2. Size of the $\lambda / 4$ impedance transformer employing the ML/ CPW composite structure and of a conventional coplanar waveguide on a silicon substrate.

\begin{tabular}{cccc}
\hline Items & $\begin{array}{c}\text { Signal line } \\
\text { Width }(\mathrm{mm})\end{array}$ & $\begin{array}{c}\text { Length } \\
(\mathrm{mm})\end{array}$ & Size $\left(\mathrm{mm}^{2}\right)$ \\
\hline \hline $\begin{array}{c}\text { Conventional } \\
\text { CPW }\end{array}$ & 1 & 0.96 & 0.96 \\
\hline $\begin{array}{c}\text { TFTL } \\
\text { ML/CPW }\end{array}$ & 0.02 & 0.5 & 0.01 \\
\hline
\end{tabular}

silicon substrate. The structure and the fabrication process for the impedance transformer in Fig. 7 are the same as those of the thin-film transmission line seen in Fig. 2. In other words, the $\mathrm{SiO}_{2}$ was deposited over the second ground plane, and the line and ground metal were placed on the top layer. The top layer ground 


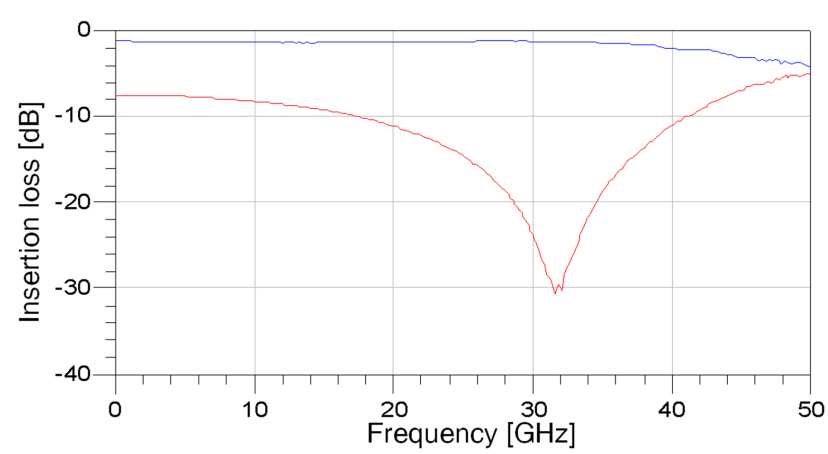

Fig. 8. Measured RF characteristics of the thin film impedance transformer employing a ML/CPW composite structure.

plane was connected to the second ground plane through the contact.

Figure7 shows a photograph of the impedance transformer. The characteristic impedance, $Z_{0}$, of the transformer was obtained using the equation $\mathrm{Z}_{\mathrm{o}}=\left(\mathrm{Z}_{\mathrm{c} 1} \cdot \mathrm{Z}_{\mathrm{c} 2}\right) 0.5$ [14], where $\mathrm{Zc1}$ and $Z_{\mathrm{c} 2}$ are the source and load impedances, respectively. For the impedance transformer, $Z_{c 1}$ and $Z_{c 2}$ are $12 \Omega$ and $27 \Omega$, respectively, and the characteristic impedance $Z_{0}$ is $18 \Omega$. The size of the proposed impedance transformers is summarized in Table 2. For comparison, the size of the impedance transformers employing a conventional coplanar waveguide was also listed on this table. As shown in this table, the thin film impedance transformer employing the ML/CPW composite structure shows a much smaller size than that of a conventional one. Specifically, the size of the impedance transformer was $0.01 \mathrm{~mm}^{2}$, which is $1.04 \%$ that of a transformer fabricated using a conventional coplanar waveguide on a silicon substrate.

Figure 8 shows the measured return loss, $S_{11}$, and the insertion loss, $S_{21}$, of the impedance transformer. In this figure, we can observe for the impedance transformer to have an excellent RF performance. Specifically, it has a return loss and insertion loss of $-31 \mathrm{~dB}$ and $-1.2 \mathrm{~dB}$, respectively, at a center frequency of 31.6 $\mathrm{GHz}$, and we can observe the return loss values to be better than $-10 \mathrm{~dB}$ from 18 to $41 \mathrm{GHz}$ with insertion loss values of $-1.7 \pm 0.4$ $\mathrm{dB}$.

\section{CONCLUSIONS}

In this work, we fabricated a TFTL (thin-film transmission line) employing a ML/CPW composite structure on a silicon substrate, and we thoroughly investigated its RF characteristics. The measured results indicated that the TFTL the proposed structure showed a periodic capacitance value higher than that of a conventional coplanar waveguide and a thin film microstrip line. Specifically, the TFTL with the proposed structure showed a capacitance of $0.88 \sim 0.93 \mathrm{pF} / \mathrm{mm}$ from 5 to $30 \mathrm{GHz}$ while the conventional coplanar waveguide and thin-film microstrip line showed capacitances of $0.13 \sim 0.17$ and $0.34 \sim 0.36 \mathrm{pF} / \mathrm{mm}$, respectively, over the same frequency range.

The improvement of the periodic capacitance of the TFTL with the proposed structure exhibited a shorter wavelength than that of a conventional coplanar waveguide and a thin-film microstrip line. Specifically, the wavelength of the TFTL with the proposed structure was of $6.26 \mathrm{~mm}$ at $10 \mathrm{GHz}$ while those of the conventional coplanar waveguide and thin-film microstrip line were 10.35 and $7.83 \mathrm{~mm}$, respectively, over the same frequency.

The TFTL with the ML/CPW composite structure at a length of $\lambda / 8$ showed a loss of less than $1.12 \mathrm{~dB}$ at up to $30 \mathrm{GHz}$, which was lower than that of a conventional coplanar waveguide. The improvement in the periodic capacitance allowed for the TFTL employing the ML/CPW composite structure to show a value in the propagation constant higher than that of a conventional coplanar waveguide and a thin film microstrip line. Specifically, the TFTL with the ML/CPW composite structure showed a $\beta$ of $0.53 \sim 2.96 \mathrm{rad} / \mathrm{mm}$ from 5 to $30 \mathrm{GHz}$ while the conventional coplanar waveguide and thin-film microstrip line showed a $\beta$ of $0.33 \sim 1.57 \mathrm{rad} / \mathrm{mm}$ and $0.4 \sim 2.27 \mathrm{rad} / \mathrm{mm}$, respectively, over the same frequency range.

In addition, the TFTL with the ML/CPW composite structure showed an effective permittivity, $\varepsilon_{\text {eff }}$, that was higher than that of a conventional coplanar waveguide and a thin-film microstrip line. The TFTL with the proposed structure showed an $\varepsilon_{\text {eff }}$ of $22.3 \sim 25.3$ from 5 to $30 \mathrm{GHz}$ while the conventional coplanar waveguide and thin-film microstrip line showed an $\varepsilon_{\text {eff }}$ of 6.28 9.87 and 13.0 14.6, respectively, over the same frequency range.

We used the TFTL employing an ML/CPW composite structure to fabricate a highly miniaturized impedance transformer on a silicon substrate. The size of the impedance transformer was of $0.01 \mathrm{~mm}^{2}$, which was only $1.04 \%$ of the size of the transformer fabricated using a conventional coplanar waveguide on a silicon substrate. For a broadband application, we could observe an excellent RF performance from the impedance transformer. The return loss and the insertion loss were of $-31 \mathrm{~dB}$ and -1.2 $\mathrm{dB}$, respectively, at a center frequency of $31.6 \mathrm{GHz}$, and we could observe return loss values better than $-10 \mathrm{~dB}$ from 18 to $41 \mathrm{GHz}$ and insertion loss values of $-1.7 \pm 0.4 \mathrm{~dB}$ in the above frequency range.

\section{ACKNOWLEDGMENTS}

This research was financially supported by the Ministry of Education, Science Technology (MEST) and National Research Foundation of Korea(NRF) through the Human Resource Training Project for Regional Innovation. This work was supported by the National Research Foundation of Korea(NRF) grant funded by the Korea government(MSIP) (2014R1A2A1A11049844).

\section{REFERENCES}

[1] D. R. Webster, G. Ataei, and D. G. Haigh, IEEE Trans. Microwave Theory Tech., 49, 328 (2001). [DOI: http://http://dx.doi. org/10.1109/22.903093].

[2] R. E. Collin, Foundation of Microwave Engineering, $2^{\text {nd }}$ ed. (McGraw-Hill, 1992).

[3] J. C. Chiu, J. M. Lin, and Y. H. Weang, IEEE Microw. Wireless Compon. Lett., 16, 449 (2006).

[4] Li, X., Y. J. Yang, L. Yang, S. X. Gong, T. Hong, X. Chen, and Y. J. Zhang, Microwave Opt. Technol. Lett., 52, 1736 (2010).

[5] T. Hirota, A. Minakawa, and M. Muraguchi, IEEE Trans. Microwave Theory Tech., 38, 270 (1991).

[6] M. C. Scardelletti, G. E. Ponchak, and T. M. Weller, IEEE Microw. Wireless Compon. Lett., 12, 6 (2002).

[7] Y. B. Park, H. N. Joh, S. H. Kim, Y. Yun, and I. H. Kang, Proc. of 10th Conference On Science and Technology (HCM city, Vietnam, 2007) p.135-138.

[8] B. C. Wadell, Transmission Line Design Handbook (Artech House, 1991).

[9] F. Schnieder, and W. Heinrich, IEEE Trans. Microwave Theory Tech., 49, 104 (2001). 
[10] F. H. M. Heilinger, M. Nagel, H. G. Roskos, H. Kurz, F. Schnieder, and W. Heinrich, IEEE MTT-S Int. Microwave Symp. Dig., 421 (1997).

[11] K. J. Son, J. H. Jeong, S. J. Han, and Y. Yun, Applied Mechanics and Materials, 442, 138 (2014).

[12] W. Chen, X. Huang, X. Ma, M. Cai, and D. Yang, $14^{\text {th }}$ Internation- al Conference on Electronic Packaging Technology, 1284 (2013).

[13] D. Kim, H. Kim, and Y. Eo, Electronics Letters, 49, 1084 (2013) [DOI: http://http://dx.doi.org/10.1049/el.2013.1444].

[14] D. M. Pozar, Microwave Engineering Reading (MA: AddisonWesley, 1990).

[15] J. R. Long, IEICE Trans. Electron., E86-C, 1022 (2003). 